\title{
Exploring Transformative Fandom to Broaden Participation in Computing
}

\author{
Brianna Dym \\ University of Colorado Boulder \\ Boulder, CO 80309, USA \\ brianna.dym@colorado.edu
}

\begin{abstract}
Broadening participation in computing is essential for involving a diversity of perspectives in creating new technologies. Despite attempts to involve diverse groups in computer science, underrepresented groups such as women; racial, gender, and sexual minorities; and people with disabilities are still under-involved in formal computing spaces such as education and industry. In contrast to examining how to bring underrepresented groups into these spaces, I propose examining examples of computational work already happening within marginalized communities, where
\end{abstract}

Permission to make digital or hard copies of all or part of this work for personal or classroom use is granted without fee provided that copies are not made or distributed for profit or commercial advantage and that copies bear this notice and the full citation on the first page. Copyrights for components of this work owned by others than the author(s) must be honored. Abstracting with credit is permitted. To copy otherwise, or republish, to post on servers or to redistribute to lists, requires prior specific permission and/or a fee. Request permissions

from Permissions@acm.org.

GROUP '20 Companion, January 6-8, 2020, Sanibel Island, FL, USA (C) 2020 Copyright is held by the owner/author(s). Publication rights licensed to ACM.

ACM 978-1-4503-6767-7/20/01\$15.00

httos://doi.ora/10.1145/3323994.3371014 participants who may be outsiders to computing culture still engage with computation to empower their own communities.

\section{Author Keywords}

Computer science education, women in computing, broadening participation, LGBTQ, fandom.

\section{ACM Classification Keywords}

- Human-centered computing Collaborative content creation

\section{Introduction}

Broadening participation in computing is essential for ensuring that diverse perspectives are involved in creating new technologies. Despite initiatives aimed at bringing diverse groups into computer science [11], underrepresented groups such as women; racial, gender, and sexual minorities; and people with disabilities are still under threat to be crowded out by social problems such as stereotype threat [12], historically white and male student populations [11], and implicit bias among authority figures [14].

Despite these challenges in formal computing spaces, computing technologies also have a history of greatly empowering marginalized and minority groups when those communities can effectively make use of that 
technology [13]. Both in the hopes of understanding systemic barriers to formal participation and investigating how to support broader engagement, my work investigates how marginalized groups who may be outsiders to computing culture are engaging with computational projects in order to empower their own communities.

\section{Background}

I situate my research within transformative fandom, where participating community members transform media by producing new creative works that draws material and inspiration from the original-e.g., fanfiction stories about the continuing adventures of Harry Potter or Captain Kirk. The role of transformative works can be to celebrate the source content, critique it, correct missteps, or propose alternative readings. Often, these works are motivated by the exclusion of certain types of characters or narratives from the underlying media. For example, fanfiction writers have written more female characters into the original Star Trek series $[1,10]$, new video game narratives that redefine diverse genders and the roles of trans and nonbinary identity in games [7], and stories that help normalize disability in comic book worlds [15] and autism in the world of Harry Potter [2]

In addition to transforming media through narrative, people in fandom also transform media through technical practice, including fanvidding, a multimodal practice that includes complex video editing communities [3], and video game modding that is often aimed at diversifying overwhelmingly male and heteronormative games [17].
Fandom participants also take part in large-scale computational projects aimed at transforming technology platforms and their own communities. Ten years ago, a group of fanfiction writers launched an open source project that resulted in an online platform that today includes over 2 million users and 5 million shared creative works: Archive of Our Own (AO3). The code that these (mostly female) developers wrote represents careful, value-based design decisions that reflect accessibility, inclusivity, and safety [8]. Moreover, during the development process, women learned to code so that they could contribute-and established a positive learning environment where they taught others as well [9]. In this remarkable example, a community needed a technology of their own-so they created it themselves, picking up new skills and bringing others into computing along the way.

Prior work examining fandom prompts the question: How do participants engaged in computational work see themselves as connected to the broader discipline of computing? How is this new type of computing community creating something different? And how might we encourage traditionally marginalized people in computing to create their own spaces and let them flourish while also transferring lessons learned to more formal computing areas?

\section{Current Research Plan}

Based on these research questions, I propose inquiring into two spaces where fans are participating in computational projects: 1) interviewing video game modding communities and, 2) observing and interviewing developers involved in building social media platforms for transformative fandom. 
Video games represent a medium that has a strong pull for encouraging people to learn computational skills, and there have been many initiatives to leverage video games into a broader interest in computer science [5]. However, one of the most important aspects of games leading to an interest in computer science is a desire to know "how things work," including breaking open the box by hacking or modding [4].

Within fandom, game modding is analogous to fanfiction; it is a way of changing or adding to an existing story [16].Video games carry the same systemic issues that other media types do that fanfiction writers typically respond to, especially from the perspective of marginalized groups like the LGBTQ community. Modding games is an especially compelling act to observe because it allows a person to physically alter the substance of a game, which is typically structured in a way that reinforces misconceptions that heterosexual, white men are the default gamer while others are secondary [6]. To better understand modding practices situated within a marginalized community, I propose conducting semi-structured interviews with game modders that share identities underrepresented in computer science.

Looking beyond modding communities, I plan to investigate the development team involved with building Pillowfort.social, a social media platform that self-identifies as a place for transformative fandom, and whose development team is clearly active within the broader fandom community. Pillowfort is an in-beta project for a social media platform that positions privacy of the user first, and draws much of its design cues from platforms that have been common social spaces for fans, including LiveJournal and Tumblr.
The development of Pillowfort also mirrors $\mathrm{AO} 3$ in that Pillowfort's design and policy are motivated in part due to fans' complaints about the design and policy decisions concerning their dominant social platform Tumblr. By interviewing site users and developers while conducting participant observation, I explore what initially drew communities to Pillowfort and why, what motivates development, and how Pillowfort has changed over time as the site grows. In examining how fandom communities undertake computational projects across multiple sites of inquiry, I hope to better understand how marginalized populations are participating in computing as well as better understand roadblocks present in traditional computing spaces. This work was primarily supported by the National Science Foundation (NSF) under award No. 1936741.

\section{How the Doctoral Colloquium Helps}

This project is in its early phase of development, meaning that any input received from senior faculty and other researchers in the field can have an invaluable impact on the scope and design of the research. While I am currently in the literature review and observation phase of research, I plan to launch interviews in Spring 2020. By connecting with other researchers who have investigated group work online, I can check my study design for potential gaps or weaknesses. Furthermore, the doctoral colloquium provides a rare opportunity to learn from others how the early phases of dissertation research might happen and what pitfalls to be aware of. Coming from a relatively new department, there are few senior PhD candidates to talk to about the dissertation process. A doctoral colloquium would help me gain perspective on the process from my peers across the discipline. 


\section{References}

[1] Camille Bacon-Smith. 1992. Enterprising Women: Television Fandom and the Creation of Popular Myth. University of Pennsylvania Press, Philadelphia, PA.

[2] Rebecca W. Black, Jonathan Alexander, Vicky Chen, and Jonathan Duarte. 2019. Representations of Autism in Online Harry Potter Fanfiction. Journal of Literacy Research 51, 1: 30-51.

[3] Francesca Coppa. 2011. An Editing Room of One's Own: Vidding as Women's Work. Camera Obscura: Feminism, Culture, and Media Studies 26, 2: 123-130.

[4] Betsy Disalvo and Amy Bruckman. 2009. Questioning video games' influence on CS interest. In Proceedings of the 4th International Conference on Foundations of Digital Games, 272-278.

[5] Betsy Disalvo, Mark Guzdail, Tom Mcklin, Charles Meadows, Kenneth Perry, Corey Steward, and Amy Bruckman. 2009. Glitch Game Testers: African American Men Breaking Open the Console. In DiGRA Conference.

[6] Brianna Dym. 2019. The Burden of Queer Love. Press Start 5, 1: 19-35.

[7] Brianna Dym, Jed R Brubaker, and Casey Fiesler. 2018. "theyre all trans sharon": Authoring Gender in Video Game Fan Fiction. Game Studies 18, 3. Retrieved from

http://gamestudies.org/1803/articles/brubaker_dym_f iesler

[8] Casey Fiesler, Shannon Morrison, and Amy S. Bruckman. 2016. An Archive of Their Own: A Case Study of Feminist $\mathrm{HCI}$ and Values in Design. In Proceedings of the 2016 CHI Conference on Human Factors in Computing Systems - CHI '16, 2574-2585. https://doi.org/10.1145/2858036.2858409

[9] Casey Fiesler, Shannon Morrison, R Benjamin Shapiro, and Amy S Bruckman. 2017. Growing Their Own:
Legitimate Peripheral Participation for Computationa Learning in an Online Fan dom Community. In Proceedings of the 2017 ACM Conference on Computer Supported Cooperative Work and Social Computing, 1375-1386.

[10] Henry Jenkins. 1988. Star Trek rerun, reread rewritten: Fan writing as textual poaching. Critical Studies in Media Communication 5, 2: 85-107.

[11] Amir Kamil, James Juett, and Andrew DeOrio. 2019 Gender-balanced TAs from an Unbalanced Student Body. In Proceedings of the 50th ACM Technical Symposium on Computer Science Education, 300-306.

[12] Amruth N. Kumar. 2012. A study of stereotype threat in computer science. In Proceedings of the 17th ACM annual conference on Innovation and technology in computer science education, 273-278.

[13] Bharat Mehra, Cecelia Merkel, and Ann Peterson Bishop. 2004. The internet for empowerment of minority and marginalized users. New Media and Society 6, 6: 781-802

[14] Elizabeth Patitsas, Michelle Craig, and Steve Easterbrook. 2014. A historical examination of the social factors affecting female participation in computing. In Proceedings of the 2014 conference on Innovation \& technology in computer science education, 111-116.

[15] Adrienne E. Raw. 2018. Normalizing Disability: Tagging and Disability Identity Construction through Marvel Cinematic Universe Fanfiction. Canadian Journal of Disability Studies 8, 2: 185-200.

[16] Sarah Stang. 2019. "This Action Will Have Consequences": Interactivity and Player Agency. Game Studies 19, 1.

[17] Tom Welch. 2018. The Affectively Necessary Labour of Queer Mods. Game Studies 18, 3. 